\title{
Exploration on the Importance of Singer's Emotion and Aesthetic Imagination in Vocal Performance
}

\author{
Zhiqiang LV \\ Hohhot Vocational College (China Nei Menggu010051)
}

\begin{abstract}
Keywords: Vocal singing; Singing emotion; Aesthetic imagination
\end{abstract}
\begin{abstract}
As the main body of vocal performance, the singer's aesthetic imagination and emotional control level in vocal performance often has a direct impact on the performance level of vocal music, as well as the audience's experience and feelings. Based on the expression mechanism of singer's emotion and aesthetic phenomenon in vocal performance, firstly, the author analyzes the function and value of singer's emotional expression in vocal performance. Secondly, it analyzes the importance of aesthetic imagination in vocal performance, and the research goal of this article is to help singer to better control their singing emotions and enhance the aesthetic imagination.

As a kind of special manifestation of culture and art, the singing of vocal music not only includes the singing skills of the singer, but also includes many aspects of the understanding of the art forms of songs and the display of humanistic connotation. In the process of singing, the singer's emotion and aesthetic imagination are often used as important qualities to measure the expressiveness of his works. Only when the above both are applied to singing activities can they ensure the perfect deduction and expression of the vocal works and can they be shocked audience's performance effect.
\end{abstract}

\section{Part One Summary of Emotion and Aesthetic Imagination of Singer in Vocal Performance}

In the process of vocal performance, according to singer's usual training skills and links, he provides the audience with a rich aesthetic experience by stimulating his emotions and using his own singing skills to perform works. In this process, the artist's emotion affects his singing performance, so emotional control is crucial to the singer. Another technical point that is easily overlooked by singers is aesthetic imagination. Most singers are often required to strengthen their skills training, including breath training and sound control skills, during routine training and learning, and by practicing some of the often-played tracks to practice empiricism, while the training in aesthetic imagination is somewhat less than adequate. In order to further analyze the importance of singer's emotion and aesthetic imagination, this article tries to elaborate from the following two aspects.

\section{Part Two The Value of Emotional Expression of Singer in Vocal Performance}

Vocal performance of singer in the emotional expression of music will often become the decisive factor in the performance of their presence, and some of the best vocal singers can always mobilize their emotions in a relatively short period of time, and then complete the emotional transmission and expression to resonate with the audiences. Accordingly, some vocal singers are always can not be perfect in terms of emotional control. Although they have excellent voice and breath control capabilities, they lack the ability of controlling emotions and often fail to achieve further development and breakthrough. Combined with the use of vocal performance skills in vocal performance, the value of vocal performance can be divided into the following aspects:

\section{Deepening the Understanding of the Work}

Different vocal music works have specific melodies and themes, which are not only full of creator's emotions, but also with the creator's environment, historical conditions and creative background that are closely related. Only through the in-depth understanding of the works can we truly understand the creative intention of the creators and give better expression to the internal emotions of the works, which plays an important role in deepening the audience's understanding of 
the works. For example, we are familiar with the "The song of the seven son", which incorporates a great deal of enthusiasm for the motherland and the hope of returning during the creative process. Therefore, singer should emphasize the ardent expectations in this regard when singing. In the process of emotional expression, people often can judge the various aspects of the work through the writing context, creative approach and structure of the work so as to enhance the effect and level of appreciation. In a good vocal works, it often has the beginning, the climax and the end, and how to finish these links by emotion is the basic skill of every vocal singer . For example, in the song "Red Flowers Blossom", because the variation of the melody is the ABA, so there should be corresponding training when dividing the emotion. The first paragraph is emotional expression of high-pitched freedom, and the second paragraph is a passionate emotion, and the third paragraph returns to the high-pitched freedom of the first paragraph. It can be seen from this that only by properly controlling the emotions can singer gain a better understanding of the control of vocal rhythm and the utilization of emotion so as to give the vocal a more unique cultural and artistic humanistic tone. This is also an important distinction between vocal music and other forms of culture and art.

\section{Better Grasping the Emotional Value of the Works}

Grasping the emotional value of the works is also a required course in the singer's emotional expression training. In the daily training process, we often ask trainers to continuously improve their emotional grasp ability, and in-depth understand the work's emotional context, and choose the corresponding emotional expression modes according to the characteristics of different works. Such as some works are sweet, and some works are unrestrained, while some works are pathetic and sad, and different works should pay attention to the choice of different emotions in singing, in order to realize its unique value. At the same time of study, the singer should also try to keep the tension, and control overall characteristics of their emotional as much as possible, in order to grasp the vocal words and sentences to let the emotional changes become more smooth and delicate and to show their singing skills but also to bring the audience a better cultural and artistic aesthetic experience.

\section{Using the Sound to Match Cleverly}

Voice is also one of the most important performance elements in vocal performance. During the singing process, the singer can attract part of the audiences through some unique musical characteristics. Different timbre not only can impress people, but also become the personal "label" of the singer in order to manifest individuality and value. With the continuous development of vocal music in our country, the current outstanding singers are endless, which is representing that different singing skills and personality singers are endless. In order to stand out in many singers, in addition to excellent singing skills and emotional expression, but also singer should learn to use of their own tonal collocation cleverly, which reflects the personal concert with other singers, which not only can enhance singer singing enthusiasm and creative desire, but also can better highlight the individual personality and value that also plays an important role in promoting.

\section{Part Three The Importance of Aesthetic Imagination in Vocal Performance}

Aesthetic imagination is often an easily neglected element in vocal performances, because the concept of aesthetic imagination is more abstract than other learning elements. It is also a slow process of increasing the singer's ability to sing. In order to better demonstrate the importance of aesthetic imagination in vocal performance, the author also tries to analyze from the following aspects.

\section{Enhancing the Imagination}

When we initially contact something, we tend to be influenced by its appearance, or we are disgusted or attracted, and the most lasting preservation of human memory is also the appearance of things. In the process of singing, we often emphasize that the singer should give the audience a "good impression". This good impression refers to the appearance of singing performance. In order to further enhance the appearance, in the complex singing environment, singers should maintain a high singing ability, and the skills and singing emotions should be transformed into a body memory, so as to achieve vocal music details more perfectly, which is nor easily exposes some of the obvious 
shortcomings. In this process, aesthetic imagination will play a decisive role. Some excellent singers can always hold their own high degree of concert status on stage through various imaginations. They can not only develop the psychological quality to the utmost, but also improve their viewing experience. This shows that aesthetic imagination has some value in vocal performance.

\section{Improving the Activity of Thinking}

Although we often say that the artistic form of music is a form of nihilism, it is made from the more rigorous rhythms and symbols. Therefore, the author believes that the combination of active thinking and scientific technology is the key to vocal singers constantly improve their ability to sing. During the process of singing, some singers ignore their subjective initiative and think that they could obtain good performance effect by practicing basic skills and "playing normally" on the stage. In essence, they have ignored the requirement of "subjectivity". In the process of singing, in order to better express the artistic conception of the work, the singer must keep the imaginary picture at all times and maintain the active thinking, so as to stimulate the audience's emotional resonance. As a result, this not only can enrich the emotional expression of the work itself, and the sublimation of the emotional mood of the song also has a catalytic effect.

\section{Enhancing Aesthetic Experience}

Aesthetic experience is often the crowning touch of what we call vocal performance. In the singing performance, the singer's psychological factors will be concentrated and enlarged, and these emotions will be delivered to each audience through the voice, so as to achieve the aesthetic art experience expression and transmission. During the singing process, the performer can directly influence the audience's inner activities by adjusting his own voice. For example, in "The Yellow River Resentment", the author can arouse the sadness of the audience with a low, slow voice and give audiences an immersive experience, which highlights the value of aesthetic experience.

\section{Conclusion}

In summary, the aesthetic imagination and emotional expression not only determine the singer's level of expression of skills, at the same time can enhance the performance of the tension and the lively atmosphere, and enhance the singing effect that has a certain role in promoting. Combined with the current application of aesthetic imagination and emotional expression in vocal performance, this article also elaborates on deepening the understanding of the work, grasping the emotion of the work, using the timbre cleverly and enhancing the imagination and enhancing the aesthetic experience. This paper can provide new ideas for enhancing vocal performance ability and emotional tension of vocal singers.

\section{References}

[1] Xiaomin Liu. The importance of the singer's emotion and aesthetic imagination in vocal music singing[J/OL].Modern Communication, 2018(2):8

[2] Jing Wu. Emotional expression and aesthetic imagination in vocal music singing[J].The Sound of the Yellow River,2016(11):101-102.

[3] Jia $\mathrm{Xu}$. An analysis of the role of emotional expression and aesthetic imagination in vocal music[J].Time and Space of Music,2015(24):85.

[4] Wei Xiong. The role of emotional expression and aesthetic imagination in vocal music[J].The Sound of the Yellow River,2013(01):102-103.

[5] Lin Lv. Study on the aesthetic imagination and emotional expression in vocal music singing[J].Academic Periodical (Journal of Shenyang Conservatory of Music),2008(03):199-202. 